\title{
Microglia mediate postoperative hippocampal inflammation and cognitive decline in mice
}

\author{
Xiaomei Feng, ${ }^{1}$ Martin Valdearcos, ${ }^{2}$ Yosuke Uchida, ${ }^{1}$ David Lutrin, ${ }^{1}$ Mervyn Maze, \\ and Suneil K. Koliwad 2,3 \\ ${ }^{1}$ Department of Anesthesia and Perioperative Care, ${ }^{2}$ The Diabetes Center, ${ }^{3}$ Department of Medicine, University of \\ California, San Francisco, San Francisco, California, USA.
}

\begin{abstract}
Surgery can induce cognitive decline, a risk that increases with advancing age. In rodents, postoperative cognitive decline (POCD) is associated with the inflammatory activation of hippocampal microglia. To examine the role of microglia in POCD, we inhibited the colonystimulating factor 1 receptor (CSF1R) in adult mice, effectively depleting CNS microglia. Surgical trauma (tibial fracture) reduced the ability of mice to remember a conditioned response learned preoperatively, a deficit more pronounced and persistent in mice with diet-induced obesity (DIO). Whereas microglial depletion by itself did not affect learning or memory, perioperative microglial depletion remarkably protected mice, including those with DIO, from POCD. This protection was associated with reduced hippocampal levels of inflammatory mediators, abrogation of hippocampal recruitment of CCR2+ leukocytes, and higher levels of circulating inflammation-resolving factors. Targeting microglia may thus be a viable strategy to mitigate the development of POCD, particularly in those with increased vulnerability.
\end{abstract}

Authorship note: XF and MV contributed equally to this work. MM and SKK contributed equally to this work.

Conflict of interest: The authors have declared that no conflict of interest exists.

Submitted: October 17, 2016 Accepted: February 21, 2017 Published: April 6, 2017

Reference information: JCI Insight. 2017;2(7):e91229. https:// doi.org/10.1172/jci.insight.91229.

\section{Introduction}

Postoperative cognitive decline (POCD) can complicate surgery and increase morbidity and mortality, especially in the elderly $(1,2)$. Efforts to identify causes of, and treatments for, POCD have focused on the innate immune response to the aseptic trauma of surgery and consequent neuroinflammation $(3,4)$. Neuroinflammation may be a common precursor of cognitive decline, as it is also implicated in more chronic forms of neurodegeneration (5). However, because the stimulus for POCD is discrete, limiting the acute neuroinflammatory impact of surgery may afford an opportunity to preserve cognitive function, thus greatly improving surgical risk-benefit.

Though rodent models suggest that hippocampal neuroinflammation contributes to POCD (6-9), how this process is triggered and orchestrated remains unclear. Initial work focused on the idea that macrophages derived from circulating monocytes can drive postoperative neuroinflammation. Evidence for this concept comes from studying monocyte chemoattractant protein 1 (MCP-1/CCL2) and its cognate receptor (CCR2), which facilitate monocyte recruitment into tissues, including the CNS, under infectious and sterile inflammatory conditions (10). For example, CCR2-expressing macrophages accumulate in the hippocampi of mice experiencing experimental surgical trauma (11). Moreover, manifestations of POCD are reduced in mice with attenuated MCP-1 expression (12). Furthermore, depleting macrophages with clodronate reduced POCD in mice (13).

There is also evidence that postoperative hippocampal neuroinflammation involves more than just blood-borne macrophages. In particular, aseptic surgical trauma in preclinical models is associated with the accumulation and potential proinflammatory activation of hippocampal cells expressing anti-ionized calcium binding adaptor molecule 1 (Iba1), which include macrophages and microglia, the CNS analogs of macrophages $(7,8,11,13-15)$. However, the importance of microglia in POCD is unclear, as is the nature of any crosstalk between microglia and $\mathrm{CCR}^{+}$immune cells that infiltrate the hippocampus through a transiently permeable blood brain barrier (BBB) (11).

Most peripheral myeloid cell types proliferate in response to multiple inputs, one of which is CSF1 (16, 17). CNS microglia, on the other hand, depend on CSF1 receptor (CSF1R) stimulation for their survival 
$(18,19)$. Indeed, germline deletion of CSF1 or the CSF1R, which responds to both CSF1 and IL-34, prevents microglia from seeding the developing brain and leads to early death in mice $(19,20)$. By contrast, inhibiting the CSF1R in adult mice depletes microglia without impacting mortality $(18,21,22)$. Here, we take advantage of this property, inhibiting the CSF1R in order to deplete $\sim 98 \%$ of microglia from the brains of adult mice $(18,22)$, and we demonstrate the impact of doing so on neuroinflammation and POCD in both healthy and vulnerable mice. Our studies reveal the critical role of microglia in orchestrating postoperative neuroinflammation and memory loss, and a heretofore-unrecognized interplay between microglia and circulating monocytes. We thus posit that microglia could be targeted to lessen POCD, as they have been targeted to limit neurodegenerative forms of cognitive decline (23).

\section{Results}

Perioperative depletion of microglia attenuates surgery-induced cognitive decline in mice. We depleted microglia from adult mice by feeding them a standard chow diet containing PLX5622, which suppresses the tyrosine kinase activity of CSF1R $(18,22)$. Consuming a diet incorporating PLX5622 for 7 days produced a profound depletion of microglia vs. control, including in the hippocampus (Figure 1, A and B, and Supplemental Figures 1, A and B; supplemental material available online with this article; https://doi. org/10.1172/jci.insight.91229DS1), consistent with prior findings (22). By contrast, PLX5622 treatment did not affect the numbers of hippocampal astrocytes (GFAP ${ }^{+}$cells), indicating specificity for microglia (Supplemental Figure 1, A and B). Control and PLX5622-treated mice were then subjected to either an aseptic tibial fracture with internal fixation or an anesthesia-matched sham procedure, after which they were assessed for markers of hippocampal and systemic inflammation, and for memory of an association learned preoperatively in a trace-fear conditioning (TFC) paradigm (Figure 1C). TFC memory assessed in rodents within days following surgery correlates well with performance in a Morris water maze, a well-validated assay of learning and memory often used in clinical models of cognitive dysfunction $(24,25)$.

Mice display a highly variable freezing response to contextual cues designed to evoke memories of TFC when tested immediately after initial associative learning (Figure 1D). Microglial depletion on its own impacted neither this learning, nor the ability of mice to freeze in response to contextual cues, an indication of their TFC memory (Figure 1D). Mice undergoing surgery, on the other hand, were markedly less responsive than sham-treated controls to cues evoking memories of preoperative TFC when tested on postoperative day 3 (Figure 1E). By comparison, mice treated perioperatively with PLX5622 retained a memory of preoperative TFC equal to that of sham-treated controls (Figure 1E). These data indicate that perioperative microglial depletion can completely prevent the development of this experimental indicator of POCD.

Microglial depletion specifically reduces surgically induced hippocampal neuroinflammation. Surgical trauma induces inflammation in brain regions involved in memory formation, such as the hippocampus (3). In control mice, surgical trauma produced an accumulation (Supplemental Figure 2, A and C) of hippocampal microglia that had transitioned from having a lacy, highly ramified morphology indicative of a quiescent state to cells with shortened processes and larger size, indicative of inflammatory activation (Supplemental Figure 2, A and D). These microglia also had reduced expression of the purinergic receptor P2Y12 and increased expression of Iba1 (Supplemental Figures 2, B and E), characteristic of a proinflammatory polarization state.

Surgical trauma also significantly increased hippocampal levels of the proinflammatory cytokines IL-6 and MCP-1 at 6 and 24 hours following surgery (Figure 2A). Of these, IL- 6 levels reached a plateau at 6 hours and fell thereafter, whereas MCP-1 levels rose progressively during the 24-hour period. PLX5622 treatment for 7 days and then perioperatively, however, dramatically lowered the postoperative hippocampal levels of both of these inflammatory mediators (Figure 2A), indicating that microglial depletion lessens postoperative hippocampal neuroinflammation.

Surgery also induced a rapid postoperative rise in hippocampal levels of the inflammatory cytokines TNF $\alpha$ and IL-1 $\beta$. However, this rise was more transient than that seen for IL- 6 and MCP-1, measurable at 6 hours but no longer at 24 hours after surgery (Supplemental Figure 2F). Regardless, perioperative PLX5622 treatment reduced the postoperative hippocampal induction of both of these mediators, as well.

By contrast, surgery increased circulating cytokine levels in both untreated and PLX5622-treated mice; any impact of PLX5622 treatment on postoperative systemic inflammation was mild as compared with the hippocampus and was totally absent for MCP-1 at 24 hours after surgery (Figures 2B and Supplemental Figure $2 \mathrm{G})$. Thus, the antiinflammatory impact of perioperative microglial depletion is relatively specific to the brain. 
A

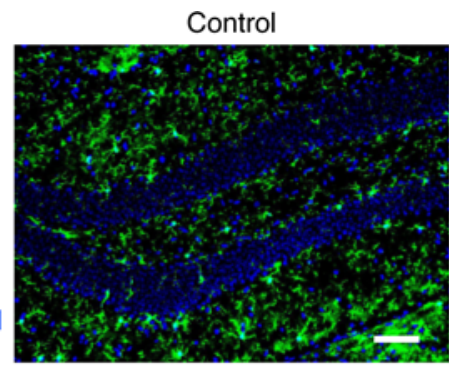

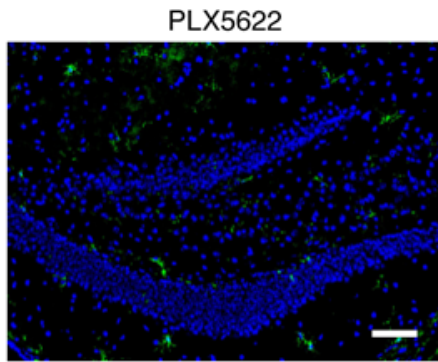

B
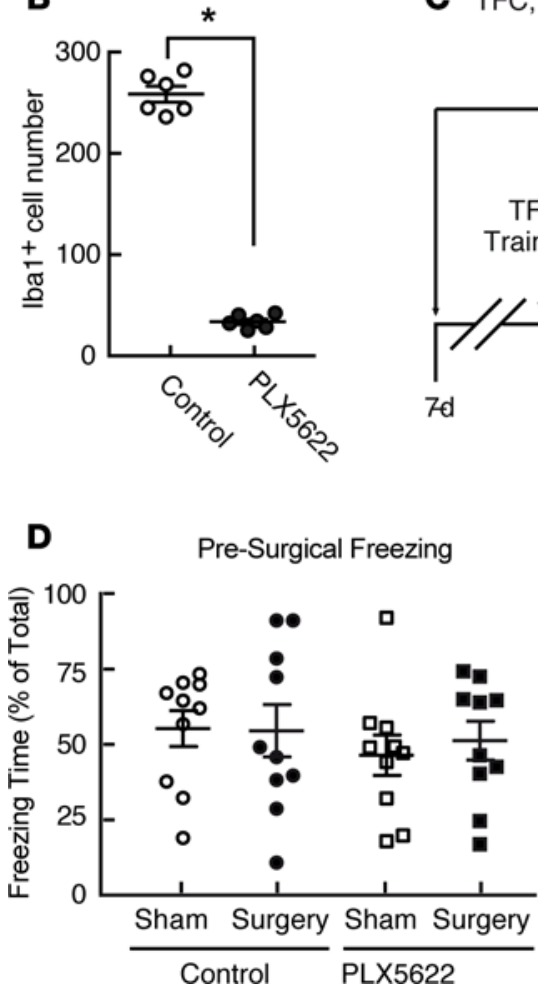

C TFC, and Systemic and Neuro-inflammation Testing Protocols

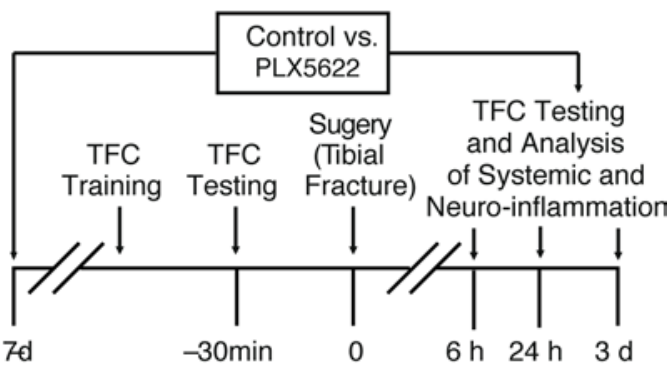

Figure 1. Perioperative microglial depletion prevents surgically induced memory loss in mice. (A) Representative hippocampal immunofluorescence images including $\mathrm{lba}^{+}{ }^{+}$cells (green) and nuclei (DAPI; blue) from mice following tibial fracture, showing marked depletion of hippocampal microglia in response to PLX5622 treatment for 7 days before surgery and 3 days following surgery. Scale bars: $50 \mathrm{~mm}$. (B) Quantification of the extent of microglial depletion in $\mathbf{A}$ ( $n=6$ /group; ${ }^{*} P<0.01$ vs. control by two-tailed Student's $t$ test). (C) Schematic, depicting the protocol for preoperative trace-fear conditioning (TFC) training, TFC testing, experimental surgery, and postoperative analyses of both hippocampal and systemic inflammation and the memory of preoperative associative learning. ( $\mathbf{D}$ and $\mathbf{E}$ ) Freezing in response to contextual cues, expressed as a percentage of total time in the testing environment prior to surgery (D) and relative to that of appropriate sham-treated controls 3 days following surgery (E). Data in D were analyzed by two-way ANOVA. Data in $\mathbf{E}$ were analyzed by two-tailed Students' $t$ test ${ }^{* * *} P=0.0002$ for surgery vs. sham-treated control). For both $\mathbf{D}$ and $\mathbf{E}, n=10 /$ group.

Microglia are required for postoperative hippocampal infiltration by BM-derived CCR2-expressing mononuclear cells. Previously, we showed that surgical trauma induces hippocampal MCP-1, the ligand for CCR2, and promotes hippocampal infiltration by CCR2-expressing monocytes (12). Moreover, treating mice with a neutralizing monoclonal antibody against HMGB1, a member of the "alarmin" family of damage-associated molecular patterns (DAMPs) that is released following trauma, is sufficient to abrogate this surgically induced increase in MCP-1, thus mitigating the neuroinflammatory impact of surgery (12). Since PLX5622 treatment protected mice from surgically induced increases in hippocampal MCP-1 levels, we assessed its effect on the recruitment of CCR2-expressing cells into the brain using mice expressing fluorescent fusion proteins of both CCR2 and fractalkine (CCR2 ${ }^{\mathrm{RFP} / \mathrm{*}}$ : $\mathrm{CX} 3 \mathrm{CR} 1^{\mathrm{GFP} /+}$ ) to distinguish monocyte-derived macrophages $\left(\mathrm{RFP}^{+}\right)$from resident microglia $\left(\mathrm{GFP}^{+}\right)$.

Whereas we did not see $\mathrm{RFP}^{+}$cells in the hippocampi of any control (sham-treated) mice (Figure 3A), $\mathrm{RFP}^{+}$cells consistently appeared in the choroid plexus, around the third ventricle, and began to enter the hippocampus within 24 hours of surgery (Figure 3B). To examine these cells further, we costained postoperative hippocampal sections for Iba1, which marks resident microglia, macrophage populations, and monocytes (Supplemental Figure 3). Focusing on the choroid plexus, we saw two populations of Iba1 ${ }^{+}$cells in this context. Some Iba $1^{+}$cells had a stellate morphology and did not coexpress CCR2 (RFP), as would be expected for resident microglia and other long-lived resident myeloid cells populations, such as perivascular and subdural macrophages. However, another population of cells coexpressed both Iba1 and RFP (Supplemental Figure 3). Probing this population revealed that, indeed, all of the CCR2-expressing $\left(\mathrm{RFP}^{+}\right.$) cells seen postoperatively in the hippocampus and associated choroid plexus also express Iba1. Moreover, these CCR2-expressing cells had either a rounded morphology or an elongated one, typical of cells in the process of vascular extravasation. Together, these findings support the concept that the CCR2-expressing cells infiltrating the hippocampus in response to surgery are monocyte derived.

Remarkably, microglial depletion abolished this surgically induced recruitment of $\mathrm{RFP}^{+}$cells (Figure $3, \mathrm{~B}$ and $\mathrm{C}$ ), indicating that microglia are responsible for the influx of circulating CCR2 ${ }^{+}$monocytes into the hippocampus following surgical trauma and suggesting that they are therefore the likely source of the chemoattractant MCP-1. By contrast, perioperative PLX5622 treatment did not deplete circulating 
A

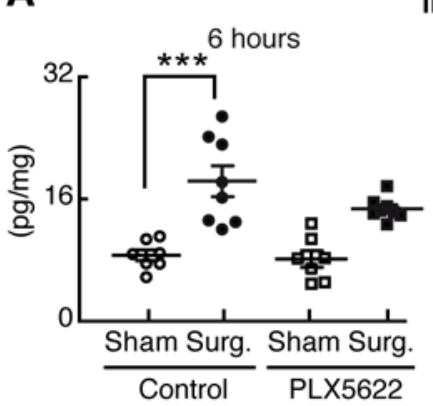

IL-6

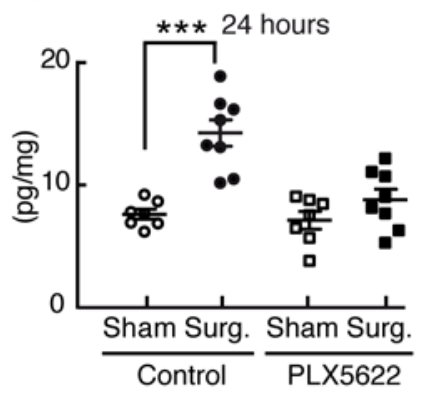

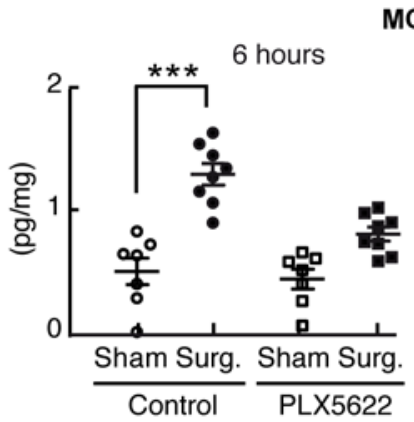

MCP-1

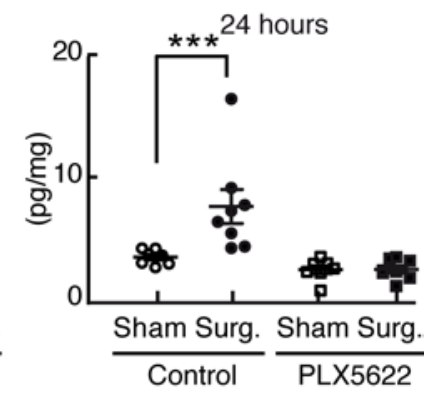

B

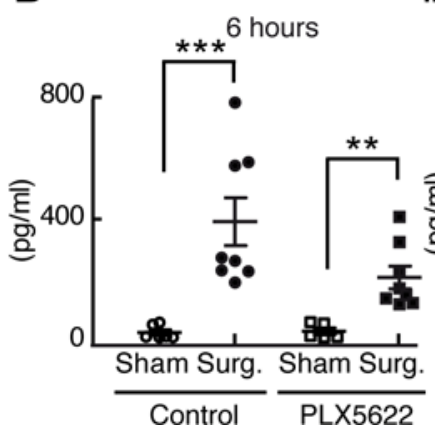

IL-6

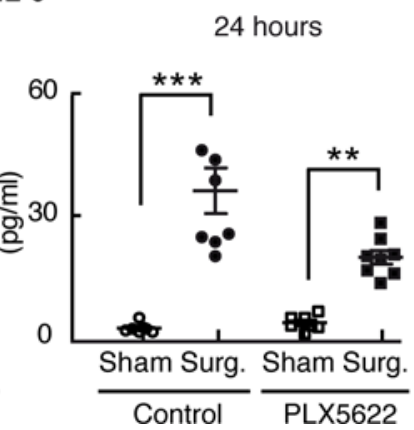

MCP-1

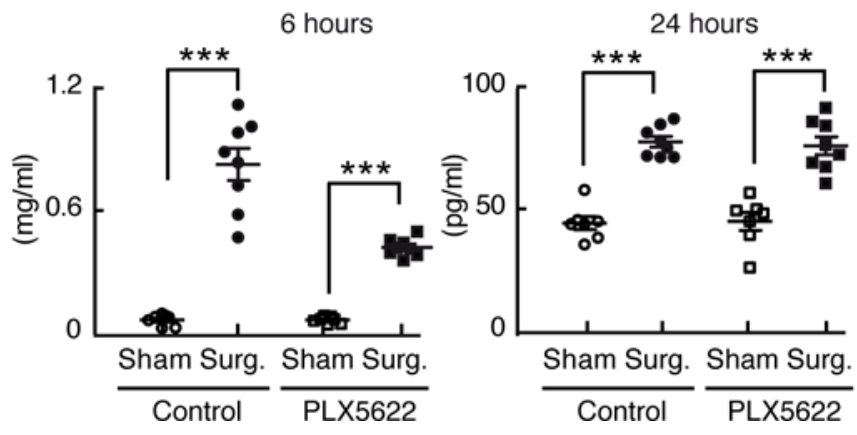

Figure 2. Perioperative microglial depletion abrogates surgically induced hippocampal inflammation. (A) Tissue ELISA, showing the rise in hippocampal levels of both IL- 6 and MCP-1 in response to surgery and the prevention of this postoperative rise by perioperative PLX5622 treatment as analyzed by twoway ANOVA ( $P=0.006$ at 24 hours for IL-6, and $P<0.01$ at both 6 and 24 hours for MCP- 1). (B) Plasma ELISA, showing the comparative lack of effect of PLX5622 treatment on postoperative plasma IL-6 and MCP-1 levels. Data were analyzed by two-way ANOVA. In all cases, $n=7-8 /$ group, and *** $P<0001$ ${ }^{* *} P<0.001$ for surgery vs. corresponding sham-treated control.

CCR2-expressing cells (Figure 3D), suggesting that PLX5622-dependent prevention of infiltration in this setting occurs through an on-target effect on microglia.

We used two other approaches to more definitively demonstrate that PLX5622 treatment abolishes postoperative infiltration of $\mathrm{CCR} 2^{+}$cells into the hippocampus through its effect on microglia, rather than through a direct effect on monocytes. We first tested the possibility that PLX5622 treatment reduces the number of infiltrative, proinflammatory monocytes. To do so, we performed multiparameter FACS analysis on circulating leukocytes from postoperative mice. Circulating CCR2 ${ }^{+}$cells were gated using markers for myeloid cells $\left(\mathrm{CD} 11 \mathrm{~b}^{+} / \mathrm{CD} 45^{+}\right)$, and the resulting pool of cells were then gated to identify individual monocyte subsets and neutrophils, respectively (Supplemental Figure 4A).

Doing so (Supplemental Figure 4B) clearly showed that PLX5622 does not deplete infiltrative proinflammatory $\left(\mathrm{Ly} 6 \mathrm{C}^{\mathrm{Hi}}\right)$ monocytes, Ly6C-intermediate $\left(\mathrm{Ly} 6 \mathrm{C}^{\mathrm{Int}}\right)$ monocytes, or neutrophils Ly6C $\mathrm{C}^{\text {int }} / \mathrm{Ly}_{6 \mathrm{G}}$ ). On the other hand, monocytes expressing little-detectable Ly6C ( $\mathrm{Ly}_{6 \mathrm{C}}{ }^{\mathrm{Lo}}$ ), which have been identified as endothelium-adherent "patrolling" monocytes (26), were depleted by $\sim 30 \%$ (Supplemental Figure 4B). However, patrolling monocytes are not implicated in tissue infiltration, suggesting strongly that PLX5622 treatment does not abolish hippocampal immune cell infiltration by depleting relevant monocyte subsets.

Next, we tested whether PLX5622 treatment impairs the ability of proinflammatory monocytes to infiltrate the brain. To do so, we injected CCR2 $2^{\mathrm{RFP} /+}$ : $\mathrm{CX} 3 \mathrm{CR} 1^{\mathrm{GFP} /+}$ mice with $\mathrm{TNF} \alpha$ by tail vein, a treatment that disrupts BBB integrity and increases CNS permeability to circulating monocytes $(27,11)$. We found that, despite depleting microglia, PLX5622 treatment did not reduce monocyte infiltration into the hippocampi of TNF $\alpha$-treated mice (Supplemental Figure 4C). This finding indicates that PLX5622 treatment does not fundamentally impair the capacity of monocytes to infiltrate the brain and supports the concept that our findings in postoperative mice result from microglial depletion, rather than a direct effect on monocytes.

Microglial depletion remains highly protective in mice with heightened vulnerability to POCD. Metabolic frailty and advanced age enhance the risk for $\operatorname{POCD}(28,29)$, and we previously modeled this heightened vulnerability in low-capacity for running (LCR) rats that spontaneously develop obesity, dyslipidemia, insulin resistance, and hypertension (30). Here, we found that mice with diet-induced obesity (DIO) 
A

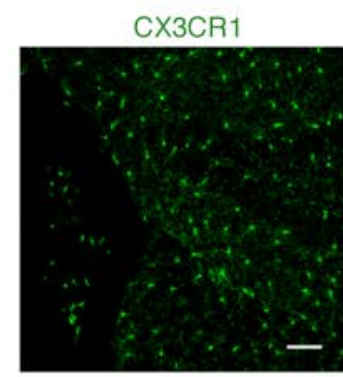

B
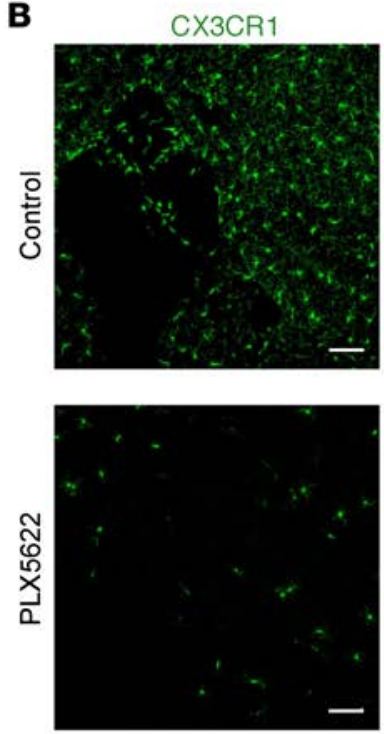

Non-surgical (sham-treated) Control

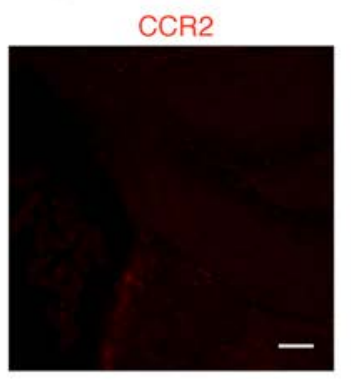

Post-Surgery
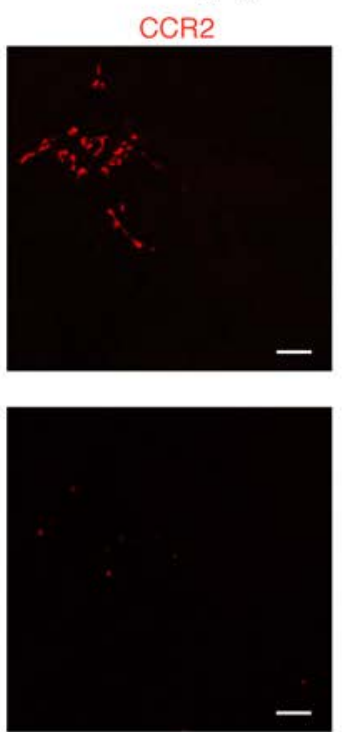

C

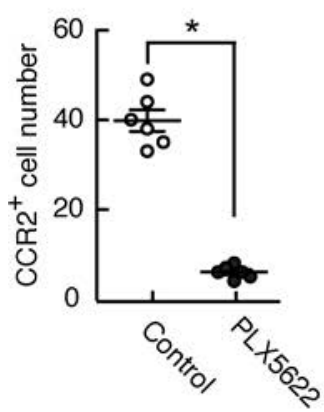

D

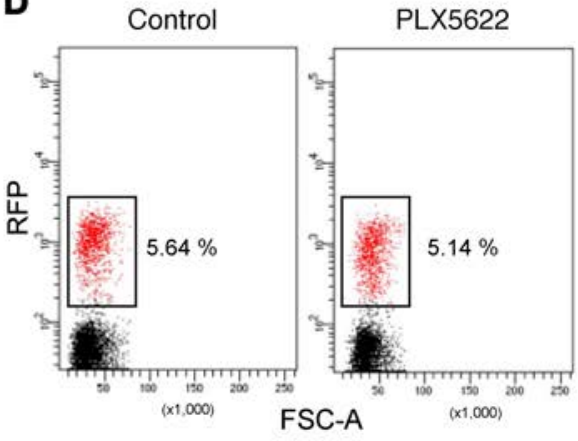

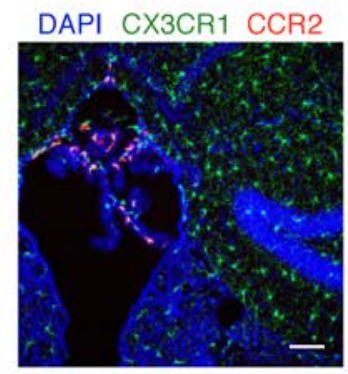
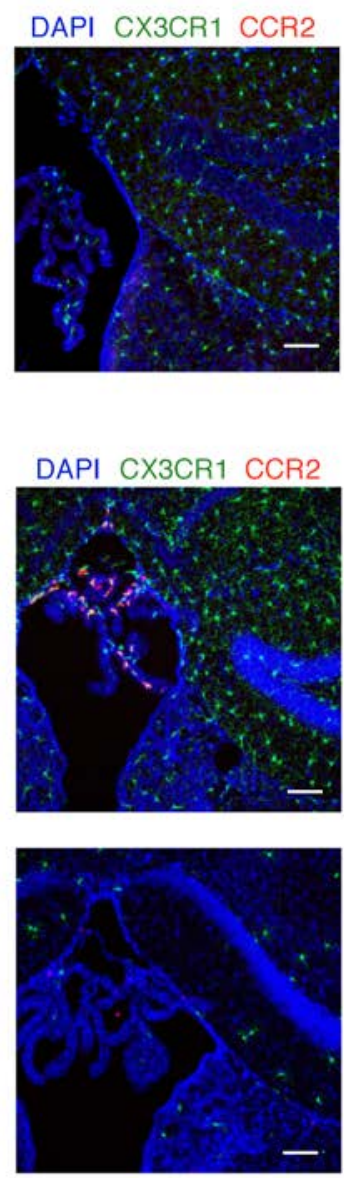

DAPI CX3CR1 CCR2

Figure 3. Perioperative PLX5622 treatment prevents surgery-induced entry of CCR1-expressing cells into the hippocampal region of the brain in mice.

(A) Representative immunofluorescence images of hippocampal sections from $\mathrm{CCR}^{\mathrm{RFP} /+}$ : $\mathrm{CX} \mathrm{C} C \mathrm{R} 1^{\mathrm{CFP} /+}$ mice $(n=4$, 3 sections per sample), showing that sham-treated mice do not have CCR2-expressing $\left(\mathrm{RFP}^{+}\right)$cells in the hippocampus or associated choroid plexus. (B) Similar images in mice 24 hours after surgery, showing that depletion of microglia (green) by perioperative PLX5622 treatment is associated with the complete disappearance of CCR2-expressing cells otherwise present in the choroid plexus and entering the hippocampus at this time point. Scale bars: $50 \mathrm{~mm}$. (C) Quantification of the prevention of surgically induced CCR2 ${ }^{\mathrm{RFP} /+}$ cell infiltration by PLX5622 treatment in mice from $\mathbf{B}(n=$ 8 mice/group; ${ }^{*} P<0.05$ vs. control by two-tailed Student's $t$ test). (D) FACS data from blood samples taken from control and PLX5622-treated mice 3 days following surgery, showing no effect of perioperative PLX5622 treatment on the number of circulating CCR2 $2^{\mathrm{RFP} /+}$ cells ( $n$ = 8 mice/group; analyzed by two-tailed Student's $t$ test).

have normal TFC memory, with the expected degree of individual variability, when tested immediately after initial learning (Figure 4A). However, like LCR rats, mice with DIO have a more pronounced and persistent loss of the freezing response to contextual testing, as performed both 3 and 7 days postoperatively, than do strainmatched healthy lean controls (Figure 4B). Remarkably, perioperative PLX5622 treatment normalized postoperative memory in mice with

DIO (Figure 4B), indicating that perioperative microglial depletion prevents postoperative memory loss, even in mice with a heightened vulnerability to POCD.

In exploring the mechanism for this vulnerability, we followed levels of eicosanoid-derived specialized proresolving mediators (SPMs) of inflammation (31); whereas surgery increased plasma levels of the SPM Lipoxin $\mathrm{A}_{4}\left(\mathrm{LXA}_{4}\right)$ in healthy mice (vs. sham controls), mice with DIO had paradoxically lower postoperative $\mathrm{LXA}_{4}$ levels than did corresponding controls (Figure 4C). These findings indicate that DIO fundamentally impairs the proresolving response to aseptic surgical trauma in mice. Consistent with this, mice with DIO had markedly higher postoperative hippocampal IL-6 levels than control mice (Figure 4D).

Remarkably, however, PLX5622 treatment prevented the postoperative abrogation in circulating LXA $_{4}$ levels otherwise seen in the context of DIO, restoring these levels to those more on par with healthy control mice. It also prevented the increase in postoperative hippocampal IL-6 levels induced by DIO (Figure 4, E and F). Together, these data indicate that depleting microglia abolishes the ability of DIO to either attenuate 
A

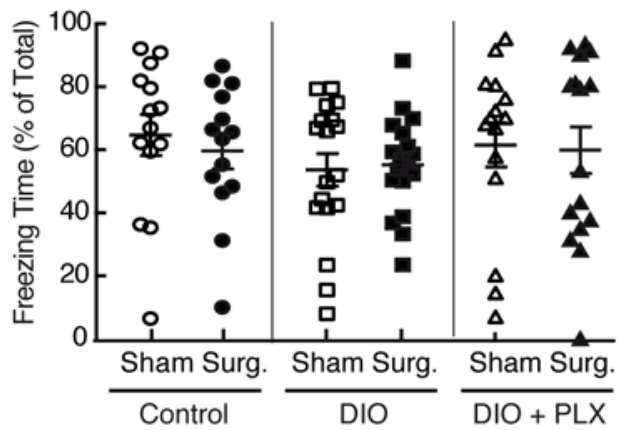

B

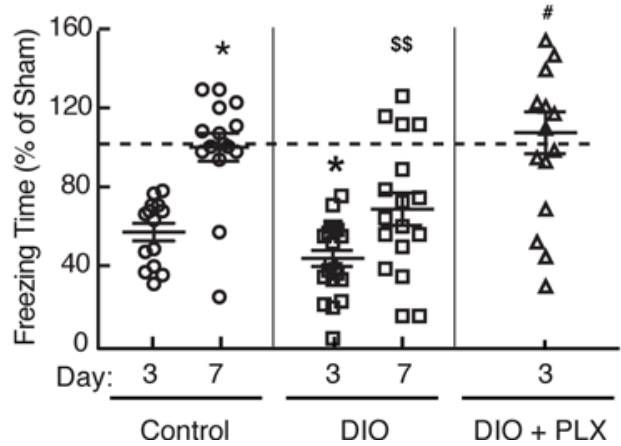

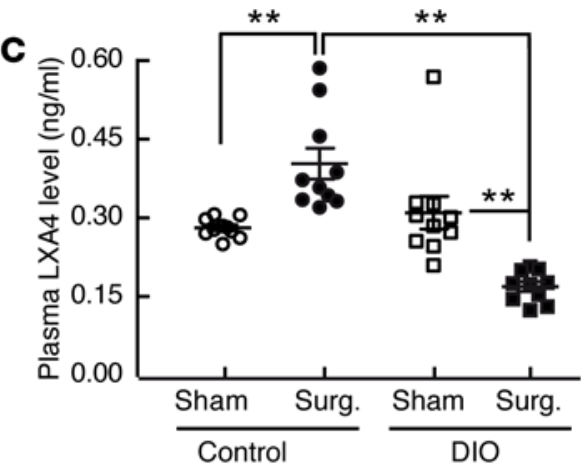

D

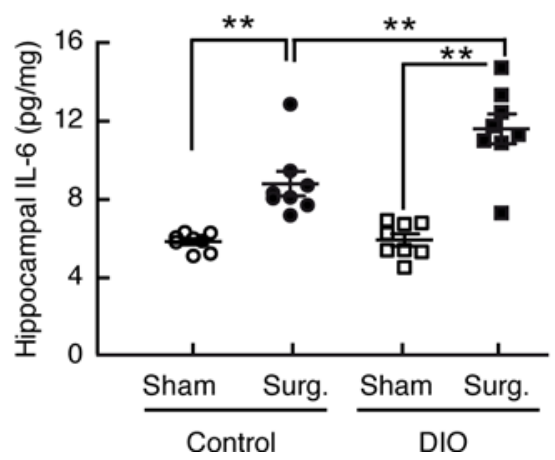

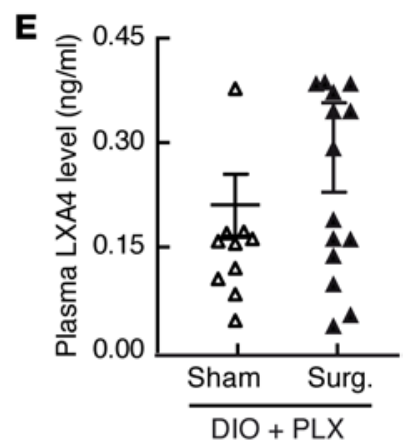

$\mathbf{F}$

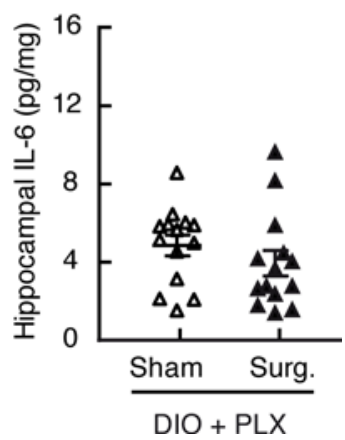

Figure 4. Perioperative microglial depletion prevents the inflammatory and cognitive consequences of surgery in mice with DIO. (A) The freezing response to preoperative TFC testing (\% of total time) of healthy (chow-fed) controls, mice with DIO, and mice with DIO treated with PLX5622 (PLX), showing no difference between groups. (B) Freezing times measured from the mice in A (\% vs. corresponding sham-treated mice) during TFC testing 3 and 7 days after surgery. Whereas the impact of surgery on TFC memory is transient and returns to sham levels by day 7 in control mice ( ${ }^{*} P<0.001$ vs. control at day 3$)$, mice with DIO have a more pronounced ( ${ }^{*} P<0.001$ vs. control at day 3$)$ and persistent $(\$ \$ P<0.001$ vs. control mice at day 7$)$ memory loss. Perioperative PLX5622 treatment protects mice with DIO from surgery induced memory loss ( ${ }^{\ddagger} P<0.005$ vs. DIO alone at day 7$)$. (C and D) Analysis of LXA ${ }_{4}$ and IL-6 levels, showing that DIO paradoxically lowers plasma LXA levels that otherwise rise by 3 days after surgery in healthy control mice (C) and potentiates the postoperative (day 3 ) rise in hippocampal IL-6 levels seen in control mice (D). (E and F) Corresponding measurements from plasma $\left(L X A_{4}\right)$ and hippocampus (IL-6), showing that perioperative PLX5622 treatment abolishes the impact of surgery on circulating LXA ${ }_{4}$ levels in mice with DIO (E) and prevents hippocampal IL-6 levels from rising in mice with DIO (F). All data are from $n=12-19$ /group and were analyzed by two-way ANOVA $(\mathbf{A}, \mathbf{C}$, and $\mathbf{D})$, one-way ANOVA (B), and Student's $t$ test $(\mathbf{E}$ and $\mathbf{F}) ;{ }^{* *} P<0.001$ for comparisons shown.

the proresolving response to surgical trauma or potentiate postoperative hippocampal inflammation. Of note, PLX5622 treatment led some mice to have relatively low circulating LXA 4 levels both under control (sham-treated) conditions and following surgery, and increased the interindividual variability in these levels (Figure 4F). However, the qualitative capacity of perioperative PLX5622 treatment to restore normal LXA responsiveness in mice with DIO was present despite this variability.

\section{Discussion}

We demonstrated that microglia are required for the development of hippocampal neuroinflammation in response to aseptic peripheral trauma. Moreover, we demonstrate that preemptively depleting mice of these cells by CSF1R inhibition prior to an experimental tibial fracture, and maintaining this depletion postoperatively, prevents not only neuroinflammation, but also the ability of surgery to impair memory.

Specifically, surgery increases hippocampal levels of proinflammatory cytokines in association with an accumulation of microglia displaying prominent features of inflammatory activation, and it induces circulating $\mathrm{CCR}^{+}$cells to enter the hippocampus. Here, we show that resident microglia are responsible for both of these processes. Indeed, perioperative microglial depletion not only abrogated the surgical induction of hippocampal cytokines, but also abolished CCR2 ${ }^{+}$cell influx into the hippocampus.

With this in mind, we were encouraged to see that, while PLX5622 treatment effectively depletes CNS microglia, it does not reduce the number of circulating CCR2 ${ }^{+}$monocytes capable of tissue infiltration $\left(\mathrm{Ly} 6 \mathrm{C}^{\mathrm{Hi}}\right)$ - consistent with prior work $(22)$ - or the number of either circulating intermediate monocytes $\left(\operatorname{Ly} 6 \mathrm{C}^{\text {Int }} / \mathrm{Ly} 6 \mathrm{G}^{\mathrm{Lo}}\right)$ or neutrophils $\left(\mathrm{Ly} 6 \mathrm{C}^{\mathrm{Int}} / \mathrm{Ly}_{16 \mathrm{G}}\right.$ ). Importantly, PLX5622 treatment did not impair the ability of monocytes to infiltrate the hippocampus in response to a nonsurgical stimulus (in this case, 
systemic TNF $\alpha$ treatment). This finding plainly shows that PLX5622 treatment does not fundamentally impair the capability of monocytes to exravasate from the bloodstream and infiltrate the brain.

On the other hand, CSF1R inhibition reduced the number of Ly6C ${ }^{\text {Lo }}$ patrolling monocytes by $\sim 30 \%$, although these monocytes are not implicated in tissue infiltration (26). It is also worth noting that $2 \%-15 \%$ of T cells may express the CSF1R, and we did not monitor the impact of CSF1R inhibition on T cell numbers. Furthermore, our examination of monocyte subsets and their infiltrative capacity does not allow us to conclude that PLX5622 has no impact on monocytes whatsoever. Indeed, it could affect monocytes in subtle or pathway-specific ways that are not evident from monitoring their numbers in the blood or the brain following surgery. Future studies should determine the dose-dependent effects of PLX5622 on monocyte function, both in vivo and in a cell-autonomous manner.

Our data support the goal of comprehensively studying the role of infiltrating immune cells in the pathogenesis of POCD. Whereas prior work implicates MCP-1-dependent macrophage recruitment in fueling POCD (12), the overall role of infiltrating cell types in this process is likely complex. For example, while Ly $6 \mathrm{C}^{\mathrm{Hi}}$ monocytes may augment the amplitude of tissue inflammation, other infiltrating cell types, including certain macrophage subsets and Tregs, may play a reparative role and work to restore hippocampal homeostasis after surgery. A combination of pharmacologic, genetic, and BM reconstitution strategies may be needed to fully assess the cell type-specific contributions of infiltrating innate and adaptive immune cells to the development of both postoperative hippocampal inflammation and POCD.

What stimulates microglial activation following surgical trauma? The alarmin HMGB1 engages circulating mononuclear cells and increases mRNA levels of the gene encoding MCP-1 (Ccl2) in the hippocampus $(4,12)$. HMGB1-activated monocytes signal through NF- $\mathrm{BB}$ and secrete TNF $\alpha$, which is implicated in disrupting BBB integrity, allowing these cells to infiltrate the brain, including the hippocampus (11). Considering this work, the data from our study supports the possibility that HMGB1 may stimulate hippocampal microglia to secrete MCP-1, enabling monocyte recruitment. These data, however, do not preclude the possibility that other mechanisms may also contribute to activating hippocampal microglia during peripheral inflammation. The surgically-induced hippocampal production of MCP-1 we observed, however, does not preclude the possibility that mechanisms other than HMGB1 may also contribute to vagal afferents that can regulate microglia (32), cytokines crossing the BBB by diffusion or active transport and regulating microglia under feedback control (33), and receptors present on endothelial cells lining the BBB or CNS-penetrating blood vessels that may control microglial polarization (34). Future work should attempt to dissect the relative contribution of these putative mechanisms in the context of surgically induced trauma.

Although the point of our cytokine measurements were to assess the ability of perioperative microglial depletion to mitigate the acute impact of surgery on hippocampal inflammation, our examination of surgically induced hippocampal inflammation also provides some insight into the kinetics with which key cytokines rise and fall. For example, IL-6 levels rose over 6 hours following surgery and then drifted slightly toward baseline. IL-1 $\beta$ and TNF $\alpha$ levels were induced even more transiently (8) and were indistinguishable from those in sham-treated controls within 24 hours of surgery. By contrast, hippocampal levels of MCP-1 rose sharply and progressively throughout the entire 24-hour period following surgery. Despite these kinetic differences, microglial depletion reduced postoperative levels of each of these factors. Thus, resident microglia orchestrate a temporally complex hippocampal cytokine response to surgical trauma.

The question of what role individual cytokines play in mediating the impact of surgery on memory loss is less clear. For example, it is quite possible that peripheral surgery alters the levels of a myriad of cytokines, chemokines, and other factors that we did not assess. Our kinetic data with respect to factors that we did measure prompt the possibility that a wide array of other molecules may also rise and fall in the hippocampus with specific kinetics after surgery. Moreover, the acute impact of surgery on cognition may not be due to any one cytokine or chemokine in isolation but may potentially involve multiple secreted factors acting in concert. Future studies should use larger scale, unbiased profiling strategies to obtain a better sense of the dynamic nature of the postoperative paracrine milieu within the hippocampus.

Beyond cognitive dysfunction, trauma also triggers fever, anorexia, fatigue, and a diminution in procreative instincts, together referred to as sickness behavior (35). Sickness behavior may protect an organism against further tissue damage by limiting excessive movement and risky behaviors in favor of rest, and by activating innate immunity. Our findings suggest that microglia may help mediate the cognitive manifestations of sickness behavior. 
When tissue healing occurs, SPMs (36) such as LXA 4 help to systemically end the initial inflammatory response at the site of insult and potentially within the brain. LCR rats do not adequately resolve postoperative inflammation and develop cognitive decline that persists for months (37). We now show that $\mathrm{LXA}_{4}$ levels do not rise appropriately following aseptic trauma in C57BL/6 mice with DIO, which also have reduced glycemic control and other features of metabolic syndrome (38). This finding indicates that, like LCR rats, mice with DIO fail to properly resolve postoperative inflammation. Moreover, mice with DIO also develop exaggerated and persistent postoperative neuroinflammation and memory loss. Our development of a mouse model of high-risk POCD will enable the use of mouse genetics to dissect how microglia, infiltrating monocytes and macrophages, and SPMs contribute to POCD.

Remarkably, PLX5622 treatment of mice with DIO not only prevented both surgically induced neuroinflammation and cognitive dysfunction, but also prevented the ability of DIO to impair the postoperative rise in circulating $\mathrm{LXA}_{4}$ levels seen in healthy lean mice without affecting inflammatory cytokine levels in the circulation. Together, these findings suggest that microglia may, at least in part, mediate the mechanism by which DIO impacts the resolution of acute inflammation. It is worth noting, though, that PLX5622 treatment itself increased the variability and tended to lower the levels of circulating LXA in sham-treated controls. One possibility for this observation is that CSF1R inhibition could independently affect the capacity of leukocytes to synthesize and/or secrete LXA 4 or other eicosanoid-derived SPMs; however, this has not been directly tested.

How might microglia regulate systemic $\mathrm{LXA}_{4}$ levels? One possibility is that acutely activated microglia could downregulate their capacity to produce $\mathrm{LXA}_{4}$. If so, the exaggerated postsurgical microglial activation seen in mice with DIO could limit the contribution of CNS-derived $\mathrm{LXA}_{4}$ to rising plasma $\mathrm{LXA}_{4}$ levels after surgery. Indeed, human microglia express key LXA 4 enzymes, including 5-lipoxygenase (5-LOX) and 5-LOX activating protein (FLAP), involved in $\mathrm{LXA}_{4}$ synthesis (39).

Another possibility is that activated microglia could, through secretion of another factor that is able to enter the circulation, indirectly reduce $\mathrm{LXA}_{4}$ production or secretion by peripheral immune cells. This possibility remains to be tested. On the other hand, preclinical models of neuroinflammation suggest that circulating $\mathrm{LXA}_{4}$ can feed back to inhibit microglial activation (40) or induce an alternatively activated (M2) state (41), suggesting the possibility of a regulatory feedback loop between LXA $_{4}$ and microglia.

Importantly, our approach avoids key off-target effects. For example, PLX5622 treatment in absence of surgery did not impact associative learning or memory. This point is important, as any acute application of CSF1R inhibition to limit POCD should not affect cognitive function on its own. The modest impact of PLX5622 treatment on circulating inflammatory markers is also potentially beneficial, as any effort to target microglia in the context of surgery or injury should preserve one's capacity to mount a systemic inflammatory response (42). Our observation that PLX5622 targets microglia while sparing proinflammatory monocytes underscores the concept that the host response to peripheral infections might remain robust in the context of perioperative CSF1R inhibition. Despite these reassuring aspects of the current study, future studies should functionally modulate microglia without depleting them in order to confirm the mechanistic interpretation of our study.

There are other aspects of our approach to microglial depletion that have translational relevance. For example, microglial numbers return to normal in less than 36 hours after stoppage of PLX5622 treatment (18), a reversibility that bodes well under clinical circumstances. Moreover, others have shown that CSF1R inhibition with PLX3397, an agent closely related to PLX5622, did not impact key elements of tibial fracture repair in relatively young rodents and actually improved aspects of fracture repair in older animals (40). This suggests that perioperative CSF1R inhibition may not fundamentally impair bone healing in the context of orthopedic surgeries.

Importantly, CSF1R inhibitors are already in clinical trials for chronic diseases ranging from cancer to inflammatory arthritis (43-45). Perioperative CSF1R inhibition would be focused and short-lived by comparison, thus minimizing patient risk. With this in mind, it is worth noting that PLX5622 has better in vivo pharmacokinetic parameters than do several commercially available CSF1R inhibitors, such as ki22027 (22). This, too, could be relevant to any downstream efforts at clinical translation. On the other hand, the relative efficacy of PLX5622 on microglial depletion in the context of obesity, especially when used in a chronic manner, is not well understood. Future studies should rigorously compare lean and progressively obese mice to specifically test this question. Our own early work, however, suggests that the ability of CSF1R inhibition to deplete microglia in mice remains substantial in the face of DIO (unpublished observation). 
It will be important to determine the durability of microglial depletion in preventing POCD over the long-term, as opposed to the early postoperative period examined here. Though work to this end is progressing, the present study does shed some light on this topic. The LCR rat model manifesting chronic POCD $(24,37)$ is similar to the DIO mouse model examined here. Both models show a more pronounced loss of TFC memory when assessed within days after surgery. Indeed, the DIO mice studied here showed a heightened (3 days) and persistent (7 days) loss of TFC memory. Acute postoperative TFC memory loss in LCR rats was predictive of persistent memory loss assessed by the Morris water maze several weeks later (24). Given that perioperative microglial depletion completely protected against the acute loss of TFC memory induced by surgery, even in mice with DIO, there is strong reason to believe that it would protect mice with DIO from POCD over the long-term, as well. However, the minimal duration of microglial depletion required to achieve such prolonged protection is unknown and, thus, must be directly tested.

Our findings suggest that postoperative hippocampal function may return to normal in healthy lean mice within days after surgery. As such, we do not posit that all mice, or all humans for that matter, are vulnerable to long-term POCD. Rather, our study highlights the concept that targeting microglia may be effective in limiting the negative impact of surgery on cognition in especially vulnerable individuals. These include surgical patients with advanced age or associated metabolic diseases that may fail to readily resolve trauma-induced inflammation, placing them at high risk for persistent postoperative cognitive impairments $(24,29,30)$. We used $\mathrm{DIO}$ as a surrogate for conditions such as advanced age in mice, as it would have been difficult to know what chronological age is best to study the impact of aging on POCD in mice without studying multiple cohorts of mice across the aging spectrum. Given the increasing size of the elderly and metabolically frail populations, including those with diabetes, our findings are noteworthy.

By identifying microglia as critical components of the process by which surgical trauma induces neuroinflammation and consequent cognitive dysfunction, we may also inform strategies to lessen cognitive decline in chronic neurodegenerative conditions (23), or we may prevent further cognitive decline when patients with preexisting neurodegenerative conditions undergo surgery.

\section{Methods}

Supplemental Methods are available online with this article.

\section{Animals}

Male C57BL/6J mice were used in all studies (Jackson Laboratories). Five-week-old mice were grouphoused with 12-hour light/dark cycles and fed standard rodent chow (control; PicoLab) or a HFD with 42\% of calories from fat (TD.09682, Envigo-Teklad) for 8 weeks. Israel Charo (J. David Gladstone Institutes, San Francisco, California, USA) provided CCR2-RFP and CX3CR - GFP mice. Microglia were depleted in all cases by feeding mice a diet containing the CSF1R inhibitor PLX5622 (Plexxikon) formulated in either a standard low-fat AIN-76A chow diet Research Diets (RD) or a high-fat Western diet (D12079B, RD) at a dose of $1.2 \mathrm{~g} / \mathrm{kg}$ for 7 days. In order to disrupt the BBB independently of surgery, mice were given recombinant murine TNF $\alpha(0.15 \mathrm{mg} / \mathrm{kg}$; Peprotech; catalog 315-01A) by i.v. injection and then studied 16 hours later. Anesthesia was with $2.1 \%$ isoflurane in $0.30 \mathrm{FiO}_{2}$.

\section{Fear-conditioning tests}

Mice adopt a characteristic "freezing" posture when anticipating an aversive stimulus, the memory of which is evoked by contextual cues related to a fear-inducing stimulus-response pairing learned previously. The percentage of time that mice spend freezing when in the presence of standardized contextual cues is thus a reliable index of their memory of an experimental bout of prior associative learning. The extent of initial learning (training) and memory of this learning (contextual testing) was recorded in a TFC chamber (Med Associates Inc.).

Training. Training consisted of two trials of tone and foot-shock pairings. Mice were placed in the TFC chamber and allowed to explore it for 100 seconds. Mice were then exposed to an auditory cue (75-80 $\mathrm{Db}, 5 \mathrm{kHz}$, conditional stimulus) for 20 seconds, followed 20 seconds later by a 2-second foot shock (0.8 mAmp; unconditional stimulus). The tone and foot-shock pairing was repeated with an intertrial interval of 100 seconds, and the mice were removed from the chamber for 30 seconds between trials.

Contextual testing. Contextual test was first performed 3 days after the training protocol. At this time, the mice were placed back into the same TFC chamber in which training had occurred previously but without any tone or shock for 5 minutes. Movement of the mice was monitored by an attached tracking 
system, and Video Freeze software recorded the time spent freezing as a fraction of total time in the chamber. Contextual testing was later repeated postoperatively as a means to quantify residual memory at time points following surgical interventions.

\section{Surgery}

Surgery was performed on mice within 30 minutes after the TFC training and consisted of an aseptic open tibial fracture with intramedullary fixation performed under general anesthesia, including $2.1 \%$ isoflurane in $0.30 \mathrm{FiO}_{2}$ and buprenorphine as previously described (1). Briefly, the left hind paw was shaved and disinfected. A median paw incision was then performed, followed by the insertion of a $0.38-\mathrm{mm}$ pin in the tibial intramedullary canal. The periosteum was then stripped and osteotomy performed. After producing the fracture, the wound was irrigated and the skin sutured with 5-0 Vicryl sutures (Ethicon); thereafter, animals were allowed to recover spontaneously from the anesthetic. During the procedure, temperature was monitored and maintained between $36^{\circ} \mathrm{C}$ and $37^{\circ} \mathrm{C}$ with the aid of warming pads (Harvard Apparatus). Analgesia (s.c., buprenorphine, $0.1 \mathrm{mg} / \mathrm{kg}$ ) was administered after anesthetic induction and before skin incision. Control (sham-treated) mice received anesthesia and analgesia.

\section{Cytokine measurement}

ELISA assays (R\&D Systems) were used to measure TNF $\alpha$, IL-1 $\beta$, IL-6, and MCP-1 levels in the plasma and hippocampus.

\section{Plasma LXA measurement}

An ELISA assay (Oxford Biochemical Research) was used to measure plasma levels of LXA , following manufacturer's instructions.

\section{Immunofluorescence histology}

WT mice and CCR2-RFP: CX3CR1-GFP mice were sacrificed and perfused with saline followed by $4 \%$ paraformaldehyde in $100 \mathrm{mM}$ phosphate buffer. Their brains were removed, postfixed in the same fixative overnight $\left(4^{\circ} \mathrm{C}\right)$, and then immersed in $30 \%$ sucrose for 48 hours. Brains were then embedded in optimal cutting temperature compound, immediately frozen on dry ice, and stored $\left(-80^{\circ} \mathrm{C}\right)$. Coronal sections (35- $\mu \mathrm{M}$ thick) were cut on a cryostat, mounted on glass slides using Vectashield antifade mounting media (Vector Labs; catalog H-1200), blocked for 1 hour with 5\% BSA (Sigma-Aldrich) in PBS (UCSF Cell Culture Facility) containing 0.1\% Triton X-100 (Fisher Scientific), and incubated with an anti-Iba1 antibody (1:500 rabbit polyclonal; Wako). Immunofluorescence was performed with Alexa Fluor 488labeled anti-rabbit DAPI Vectashield solution (Vector Labs) to identify cell nuclei. Images were acquired by confocal laser-scanning microscopy (Leica TCS SP5).

\section{Image Analyses}

$\mathrm{CX}_{3} \mathrm{CR} 1^{+}$and $\mathrm{Iba}^{+}$cells in hippocampal sections were counted manually by visual inspection of anatomically matched photomicrographs, with an average taken from four sequential sections per mouse. Cells were analyzed for size by Image J software using a thresholding protocol, followed by densitometric quantification. Iba1 fluorescence intensity was similarly measured with Image J software from images captured using identical exposure times that also avoided saturating the pixel intensities.

\section{Flow cytometry}

To analyze whether, in addition to depleting microglia, PLX5622 treatment reduces the total number of circulating CCR2-expressing cells, blood samples were taken from drug-treated and control CCR2RFP: CX3CR1-GFP mice. After rbc lysis (RBC lysis buffer, eBioscience), plasma leukocyte single cell suspensions were acquired on a BD LSR II flow cytometer to measure $\mathrm{RFP}^{+}$cells and were analyzed with BD FACSDiva v6 software.

\section{Statistics}

Data are expressed as means \pm SEM. Statistical analyses were performed using the Prism 5.01 (GraphPad Software Inc.) statistical package and Stata 11.2 software (StataCorp). We performed two-tailed Student's 
$t$ tests for single comparisons and ANOVA (one-way and two-way) in the context of multiple comparisons, as appropriate (see figure legends), with post hoc Newman-Keuls testing following ANOVA analysis of nonparametric samples. We tested for normal distribution of the data with the d'Agostino and Pearson omnibus test and for equality of variances with the F-test. Two-way ANOVA was specifically used whenever there was a possibility of interaction between independent treatment variables and was performed prior to performing individual pairwise comparisons. In each case, $P<0.05$ was considered significant.

\section{Study approval}

All experiments were approved by the Institutional Animal Care and Use Committee at the University of California San Francisco (AN111420-02 and AN097767-03) and adhered to the highest standards of animal care.

\section{Author contributions}

$\mathrm{XF}$ and MV contributed equally to designing and conducting relevant experiments, analyzing data, preparing figures, and drafting the manuscript. YU and DL assisted in conducting relevant experiments. MM and SKK contributed equally to conceiving of the studies, supervising their design and completion, and writing the final manuscript. SKK prepared the final figures. MM's laboratory conducted the neurocognitive testing in mice, and SKK's laboratory performed all fluorescence microscopy.

\section{Acknowledgments}

We thank Plexxikon and Brian West for PLX5622, Israel Charo for CCR2 ${ }^{\mathrm{RFP} /+}$ CX3CR1 ${ }^{\mathrm{GFP} /+}$ mice, David Julius for the P2Y12 antibody, the UCSF Diabetes Center FACS Core (NIDDK P30 DK063720), and the UCSF Center for Cerebrovascular Research. This study was funded by NIH grants: R01 DK103175-02 (to SKK) and R01 GM104194 (to MM).

Address correspondence to: Suneil K. Koliwad, Diabetes Center, University of California, San Francisco, S1230 Medical Sciences; 513 Parnassus Avenue, San Francisco, California 94143, USA. Phone: 415.479.9623; E-mail: skoliwad@diabetes.ucsf.edu. Or to: Mervyn Maze, Department of Anesthesia and Perioperative Care, University of California, San Francisco, 1001 Potrero Avenue, Box 1363, San Francisco, California 94143, USA. Phone: 415.206.8906; E-mail: mervyn.maze@ucsf.edu.

1. Steinmetz J, Christensen KB, Lund T, Lohse N, Rasmussen LS, ISPOCD Group. Long-term consequences of postoperative cognitive dysfunction. Anesthesiology. 2009;110(3):548-555.

2. Crocker E, et al. Long-Term Effects of Postoperative Delirium in Patients Undergoing Cardiac Operation: A Systematic Review. Ann Thorac Surg. 2016;102(4):1391-1399.

3. Vacas S, Degos V, Feng X, Maze M. The neuroinflammatory response of postoperative cognitive decline. Br Med Bull. 2013;106:161-178.

4. Hirsch J, et al. Perioperative cerebrospinal fluid and plasma inflammatory markers after orthopedic surgery. J Neuroinflammation. 2016;13(1):211

5. Ransohoff RM. How neuroinflammation contributes to neurodegeneration. Science. 2016;353(6301):777-783

6. Wan Y, Xu J, Ma D, Zeng Y, Cibelli M, Maze M. Postoperative impairment of cognitive function in rats: a possible role for cytokine-mediated inflammation in the hippocampus. Anesthesiology. 2007;106(3):436-443.

7. Cibelli M, et al. Role of interleukin-1beta in postoperative cognitive dysfunction. Ann Neurol. 2010;68(3):360-368.

8. Terrando N, Monaco C, Ma D, Foxwell BM, Feldmann M, Maze M. Tumor necrosis factor-alpha triggers a cytokine cascade yielding postoperative cognitive decline. Proc Natl Acad Sci USA. 2010;107(47):20518-20522.

9. Rosczyk HA, Sparkman NL, Johnson RW. Neuroinflammation and cognitive function in aged mice following minor surgery. Exp Gerontol. 2008;43(9):840-846.

10. O'Connor T, Borsig L, Heikenwalder M. CCL2-CCR2 Signaling in Disease Pathogenesis. Endocr Metab Immune Disord Drug Targets. 2015;15(2):105-118.

11. Terrando N, et al. Resolving postoperative neuroinflammation and cognitive decline. Ann Neurol. 2011;70(6):986-995.

12. Vacas S, Degos V, Tracey KJ, Maze M. High-mobility group box 1 protein initiates postoperative cognitive decline by engaging bone marrow-derived macrophages. Anesthesiology. 2014;120(5):1160-1167.

13. Degos V, et al. Depletion of bone marrow-derived macrophages perturbs the innate immune response to surgery and reduces postoperative memory dysfunction. Anesthesiology. 2013;118(3):527-536

14. Butovsky $\mathrm{O}$, et al. Identification of a unique TGF- $\beta$-dependent molecular and functional signature in microglia. Nat Neurosci. 2014;17(1):131-143

15. Valdearcos M, Xu AW, Koliwad SK. Hypothalamic inflammation in the control of metabolic function. Annu Rev Physiol. 2015;77:131-160. 
16. McGrath KE, Frame JM, Palis J. Early hematopoiesis and macrophage development. Semin Immunol. 2015;27(6):379-387.

17. Li J, Chen K, Zhu L, Pollard JW. Conditional deletion of the colony stimulating factor-1 receptor (c-fms proto-oncogene) in mice. Genesis. 2006;44(7):328-335.

18. Elmore MR, et al. Colony-stimulating factor 1 receptor signaling is necessary for microglia viability, unmasking a microglia progenitor cell in the adult brain. Neuron. 2014;82(2):380-397.

19. Erblich B, Zhu L, Etgen AM, Dobrenis K, Pollard JW. Absence of colony stimulation factor-1 receptor results in loss of microglia, disrupted brain development and olfactory deficits. PLOS ONE. 2011;6(10):e26317.

20. Ginhoux F, et al. Fate mapping analysis reveals that adult microglia derive from primitive macrophages. Science. 2010;330(6005):841-845.

21. Dagher NN, et al. Colony-stimulating factor 1 receptor inhibition prevents microglial plaque association and improves cognition in 3xTg-AD mice. J Neuroinflammation. 2015;12:139.

22. Valdearcos M, Robblee MM, Benjamin DI, Nomura DK, Xu AW, Koliwad SK. Microglia dictate the impact of saturated fat consumption on hypothalamic inflammation and neuronal function. Cell Rep. 2014;9(6):2124-2138.

23. Spangenberg EE, Green KN. Inflammation in Alzheimer's disease: Lessons learned from microglia-depletion models. Brain Behav Immun. 2017;61:1-11.

24. Feng X, et al. Surgery results in exaggerated and persistent cognitive decline in a rat model of the Metabolic Syndrome. Anesthesiology. 2013;118(5):1098-1105.

25. Poulos AM, et al. Conditioning- and time-dependent increases in context fear and generalization. Learn Mem. 2016;23(7):379-385.

26. Carlin LM, et al. Nr4a1-dependent Ly6C(low) monocytes monitor endothelial cells and orchestrate their disposal. Cell. 2013;153(2):362-375.

27. Sharief MK, Thompson EJ. In vivo relationship of tumor necrosis factor-alpha to blood-brain barrier damage in patients with active multiple sclerosis. J Neuroimmunol. 1992;38(1-2):27-33.

28. Hudetz JA, Patterson KM, Iqbal Z, Gandhi SD, Pagel PS. Metabolic syndrome exacerbates short-term postoperative cognitive dysfunction in patients undergoing cardiac surgery: results of a pilot study. J Cardiothorac Vasc Anesth. 2011;25(2):282-287.

29. Monk TG, et al. Predictors of cognitive dysfunction after major noncardiac surgery. Anesthesiology. 2008;108(1):18-30.

30. Werner S, Grose R. Regulation of wound healing by growth factors and cytokines. Physiol Rev. 2003;83(3):835-870.

31. Shinohara M, Serhan CN. Novel Endogenous Proresolving Molecules:Essential Fatty Acid-Derived and Gaseous Mediators in the Resolution of Inflammation. J Atheroscler Thromb. 2016;23(6):655-664

32. Zhu YJ, Peng K, Meng XW, Ji FH. Attenuation of neuroinflammation by dexmedetomidine is associated with activation of a cholinergic anti-inflammatory pathway in a rat tibial fracture model. Brain Res. 2016;1644:1-8.

33. Hanisch UK. Microglia as a source and target of cytokines. Glia. 2002;40(2):140-155.

34. Langford D, Masliah E. Crosstalk between components of the blood brain barrier and cells of the CNS in microglial activation in AIDS. Brain Pathol. 2001;11(3):306-312.

35. Dantzer R, O'Connor JC, Freund GG, Johnson RW, Kelley KW. From inflammation to sickness and depression: when the immune system subjugates the brain. Nat Rev Neurosci. 2008;9(1):46-56.

36. Spite M, Clària J, Serhan CN. Resolvins, specialized proresolving lipid mediators, and their potential roles in metabolic diseases Cell Metab. 2014;19(1):21-36.

37. Su X, et al. Dysfunction of inflammation-resolving pathways is associated with exaggerated postoperative cognitive decline in a rat model of the metabolic syndrome. Mol Med. 2013;18:1481-1490.

38. Fontaine DA, Davis DB. Attention to Background Strain Is Essential for Metabolic Research: C57BL/6 and the International Knockout Mouse Consortium. Diabetes. 2016;65(1):25-33.

39. Klegeris A, McGeer PL. Toxicity of human monocytic THP-1 cells and microglia toward SH-SY5Y neuroblastoma cells is reduced by inhibitors of 5-lipoxygenase and its activating protein FLAP. J Leukoc Biol. 2003;73(3):369-378.

40. Martini AC, et al. Lipoxin A4 inhibits microglial activation and reduces neuroinflammation and neuropathic pain after spinal cord hemisection. J Neuroinflammation. 2016;13(1):75.

41. Medeiros R, et al. Aspirin-triggered lipoxin A4 stimulates alternative activation of microglia and reduces Alzheimer disease-like pathology in mice. Am J Pathol. 2013;182(5):1780-1789.

42. Slade Shantz JA, Yu YY, Andres W, Miclau T, Marcucio R. Modulation of macrophage activity during fracture repair has differential effects in young adult and elderly mice. J Orthop Trauma. 2014;28 Suppl 1:S10-S14.

43. Butowski N, et al. Orally administered colony stimulating factor 1 receptor inhibitor PLX3397 in recurrent glioblastoma: an Ivy Foundation Early Phase Clinical Trials Consortium phase II study. Neuro-oncology. 2016;18(4):557-564.

44. Tap WD, et al. Structure-Guided Blockade of CSF1R Kinase in Tenosynovial Giant-Cell Tumor. N Engl J Med. 2015;373(5):428-437.

45. Hamilton JA, Cook AD, Tak PP. Anti-colony-stimulating factor therapies for inflammatory and autoimmune diseases. Nat Rev Drug Discov. 2016;16(1):53-70. 